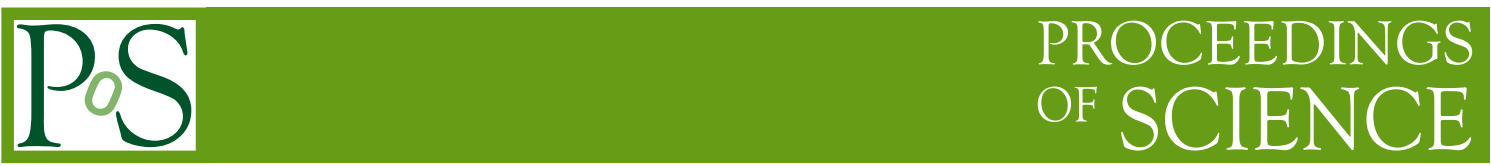

\title{
Modification of Meson Properties in External Field
}

\section{Peter Filip*}

Institute of Physics, Slovak Academy of Sciences, Dúbravská cesta 9, Bratislava 82103, Slovakia

E-mail: peter.filip@savba.sk

It is suggested, that hadron decay properties may be influenced due to the interaction of charges and magnetic moments of hadrons and constituent quarks with external electromagnetic fields. In particular, strong decays $D^{*} \rightarrow D \pi, K^{*}(892) \rightarrow K \pi, \phi \rightarrow K^{+} K^{-}$and $\Delta(1232) \rightarrow p \pi$ may become significantly modified in static magnetic field $B=10^{13}-10^{15} \mathrm{~T}$. Due to the increasing energy of the lowest $(n=0)$ Landau level of charged mesons, decay phase-space is reduced for specific channels, and isospin conservation rules become violated. In the case of $\rho^{0} \rightarrow \pi^{+} \pi^{-}$ decay suppression, enhanced production of dileptons and photons can occur. Moreover, internal wave function of quarkonium vector mesons $\Upsilon(n s)$ and $\Psi(n s)$ gets modified in the magnetic field: quantum superposition of para $(J=0)$ and ortho $\left(J=1, s_{z}=0\right)$ states becomes the eigenstate of $(c \bar{c})$ and $(b \bar{b})$ vector mesons. In a sufficiently strong electric field a superposition of $J / \Psi$ or $\Upsilon(n s)$ states with $\chi_{c}(1 P)$ or $\chi_{b}(n P)$ mesons can occur. We conjecture, that decay properties and production cross sections of Quarkonium vector mesons may thus become affected by strong electromagnetic fields created in heavy ion collision experiments at RHIC and LHC colliders.

The European Physical Society Conference on High Energy Physics

22-29 July 2015

Vienna, Austria

${ }^{*}$ Speaker. 


\section{Introduction}

Influence of electromagnetic fields on hadron properties is usually assumed to be negligible. However, in the case when external field strength becomes sufficiently large (e.g. $B \approx 10^{14} \mathrm{~T}$ ), magnetic dipole interaction of $(J \neq 0)$ hadrons $\Delta E=-\vec{\mu}_{N} \vec{B}$ moves into $\mathrm{MeV}$ range (here nuclear magneton $\mu_{N}=3.15 \cdot 10^{-14} \mathrm{MeV} / \mathrm{T}$ ) and decay properties of hadrons can be modified. It has been pointed out [1], that due to increasing energy of the lowest $(n=0)$ Landau level [2] of charged mesons in magnetic field, $\rho \rightarrow \pi \pi$ decays become suppressed and the lifetime of $\rho(770)$ mesons can be prolonged [1]. Suppression of $\rho^{0} \rightarrow \pi^{+} \pi^{-}$channel may also enhance photon and dilepton production from $\rho^{0}(770)$ decays [3], which can have interesting consequences in heavy ion collisions. In the following sections we discuss and study qualitatively the behavior of $K^{*}, D^{*}$ and $\phi(1020)$ vector mesons in strong magnetic fields and we consider also $\Delta^{0}$ and $\Delta^{++}$decays.

Additionally, we suggest that internal wavefunction of Quarkonium $(c \bar{c})$ and $(b \bar{b})$ vector mesons becomes modified in the external electromagnetic field, which can influence decay branching ratios and production cross sections of $\Upsilon(n s)$ and $J / \Psi(n s)$ states.

\section{Strong Decays of Vector Mesons in Magnetic Field}

Within MIT bag model [4], strong decay of hadron can be understood as splitting of hadronic "Bag" volume [5] due to its own quantum quadrupole vibrations. Formation of two new hadrons is accompanied by $q-\bar{q}$ quark pair creation [6] in $J^{P C}=0^{++}$quantum state. Possible influence of magnetic field [7] on such complicated QCD (string breaking) process will be neglected here and we rather concentrate on the phase-space and kinematics effects.

$\mathrm{E}[\mathrm{MeV}]$

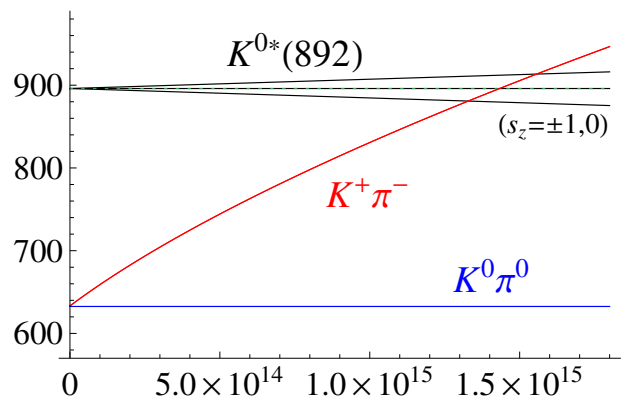

$[\mathrm{MeV}]$

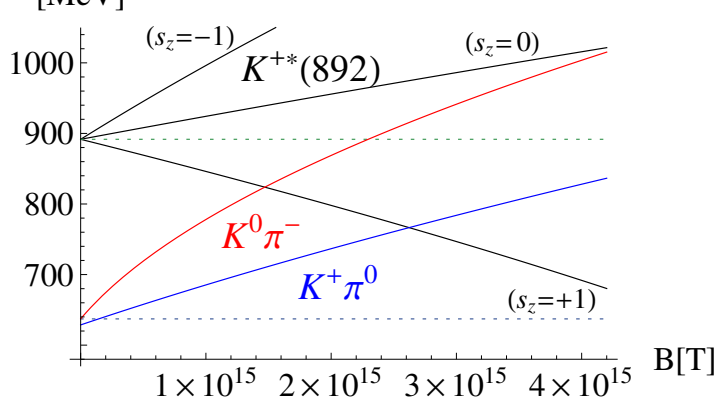

Figure 1: Triplet splitting of $K^{0 *}$ and $K^{+*}$ vector mesons (we use $\mu_{K^{0 *}}=-0.36 \mu_{N}$ and $\mu_{K^{+*}}=2.46 \mu_{N}$ ) in magnetic field $B<4 \cdot 10^{15} \mathrm{~T}$, together with the lowest possible energy $\left(p_{z}=0\right)$ of decay products $(K+\pi)$.

Energy of charged particles in the magnetic field is quantized, and the lowest possible $(n=0)$ Landau energy level of pseudoscalar mesons increases with magnetic field [1] as $E^{2}[B]=m^{2}+|Q| B$ (see Fig.1). Decay momentum $p_{z}[B]$ and decay phase-space in the magnetic field are thus modified. For $J>0$ hadron of mass $\tilde{M}=E\left[B, s_{z}\right] / c^{2}$ decaying at rest into two products with masses $m_{1}, m_{2}$ one can write (using $\Sigma^{2} m_{12}=\left(m_{1}+m_{2}\right)^{2}$ and $\left.\Delta^{2} m_{12}=\left(m_{1}-m_{2}\right)^{2}\right)$

$$
p_{z}[B]=\frac{\sqrt{\left(\tilde{M}^{2}-\Sigma^{2} m_{12}\right)\left(\tilde{M}^{2}-\Delta^{2} m_{12}\right)}}{2 \tilde{M}}, \quad E\left[B, s_{z}\right]=\sqrt{M^{2}+|Q| B-2 Q B s_{z}}+\Delta E\left[B, s_{z}\right]
$$


where $\Delta E\left[B, s_{z}\right]$ is anomalous magnetic moment interaction [3,8] of decaying particle (we neglect polarizability of hadrons here). Magnitude of decay momentum $p_{z}[B]$ is modified in magnetic field, due to rising effective masses $m_{1}, m_{2}$ of spinless charged decay products: $m_{i}[B]=\left(m_{i}^{2}+|Q| B\right)^{1 / 2}$.

Angular momentum $(J=1)$ of decaying vector meson is taken away by $L=1$ orbital momentum of two spinless pseudoscalar mesons, created in $P$-wave quantum state. In such case, partial decay width of strong decay process $R \rightarrow h+h^{\prime}$ can be written as [9]

$$
\Gamma=\tilde{m}\left|A_{h h^{\prime}}^{R}\right|^{2}(p / \tilde{m})^{2 L}(p / M) \quad \longrightarrow \quad \Gamma(p) \approx\left|A_{h h^{\prime}}^{R}\right|^{2} p^{2 L+1}
$$

where $A_{h h^{\prime}}^{R}$ is the amplitude determined by SU(3)-invariant rules of QCD, $M$ is the mass of decaying hadron (resonance), $p$ is decay momentum of products $\left(h\right.$ and $\left.h^{\prime}\right)$, and $\tilde{m} \approx 1 \mathrm{GeV} / c^{2}$ is effective mass parameter [9]. Barrier factor $(p / \tilde{m})^{2 L}$ introduces enhanced sensitivity of decay width $\Gamma(p)$ to decay momentum (phase-space) for $P$ and $D$ - wave case $(L=1,2)$. One has $\Gamma(p) \approx\left|A_{h h^{\prime}}^{R}\right|^{2} p^{3}$ for $P$-wave decay $(L=1)$ of vector mesons ${ }^{1}[10]$.

The same ( $P$-wave) behavior applies also [11] to strong decays of $(J=3 / 2)$ resonances $\Sigma^{*}$, $\Delta(1232)$, and $\Xi^{*}$. One can estimate $\Gamma[B]$ decay width as $\Gamma[B] \leq \Gamma[0]\left(p^{3}[B] / p^{3}[0]\right)$ in the magnetic field (where " $\leq$ " accounts for other external field effects [7]).

$\Gamma_{s_{z}}[\mathrm{MeV}]$

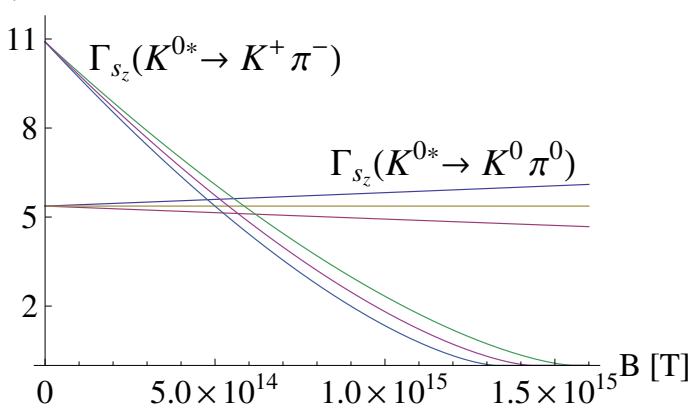

$\tau_{K^{*}}[\mathrm{fm} / \mathrm{c}]$

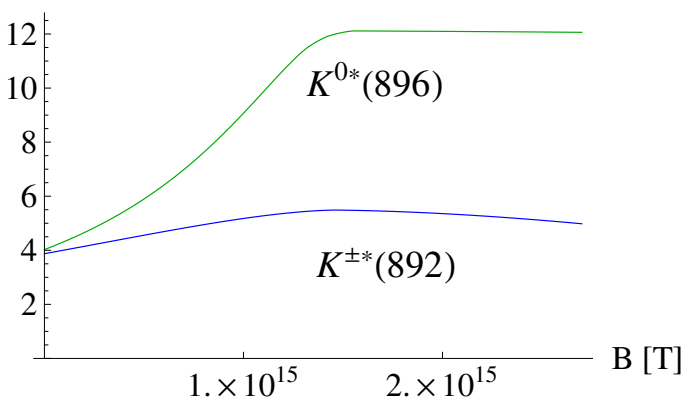

Figure 2: Partial decay widths $\Gamma_{s_{z}}[B]$ for $K^{* 0}(892)$ meson substates $\left(s_{z}= \pm 1,0\right)$ in static magnetic field $B<2 \cdot 10^{15} \mathrm{~T}$ and total lifetime $\tau_{K^{*}}$ of $K^{* 0}$ and $K^{ \pm *}$ mesons estimated according to Equation (2.3).

Partial decay widths and the lifetime of mesons in magnetic field can thus be expressed as

$$
\tau[B]=1 / \Gamma_{\text {tot }}[B], \quad \Gamma_{\text {tot }}[B]=\Gamma_{o}+\sum_{s_{z}} \Gamma_{s_{z}}[B], \quad \Gamma_{s_{z}}[B]=\frac{p_{s_{z}}^{3}[B]}{p_{s_{z}}^{3}[0]} \Gamma_{s_{z}}[0]
$$

where $\Gamma_{o}$ is field-independent part of total decay width, and $\Gamma_{s_{z}}$ are partial decay widths (see Fig. 2) for $s_{z}$ substates of $(J>0)$ meson decaying into two pseudoscalar mesons with decay momenta $p_{z}[B]$ determined by Equation (2.1), using $\tilde{M}=M_{s_{z}}[B]=E\left[B, s_{z}\right] / c^{2}$.

Lifetimes $\tau[B]$ of $K^{ \pm *}, K^{0 *}$ calculated using Equation 2.3 are shown in Figure 2 (right panel). The lifetime of $K^{ \pm *}$ remains almost unchanged, while $K^{0 *}$ lifetime increases by factor 3 in magnetic field $B>1.5 \cdot 10^{15} \mathrm{~T}$. One may also expect tensor polarization of $K^{ \pm *}$ mesons [3] to be generated in relativistic heavy ion collisions, if extremal magnetic fields [12] remain in the volume of expanding QCD matter for a long enough time.

\footnotetext{
${ }^{1}$ Decay widths $\Gamma_{ \pm}, \Gamma_{00}$ for $\phi(1020) \rightarrow K^{+} K^{-}$and $K^{0} \bar{K}^{0}$ channels [13] are different due to $p_{ \pm}^{3} / p_{00}^{3}$ ratio $\approx 1.5$.
} 
In Figure 3, we show energy of $(J=1)$ vector mesons $D^{0 *}(2007)$ and $D^{*+}(2010)$ in magnetic field $B \approx 10^{14} \mathrm{~T}$, and of their decay products $(D+\pi)$ evaluated according to approximation of Eq.(2.1): $E\left[B, s_{z}\right] \simeq m+e B / 2 m-\left(s_{z}\right) \mu^{*} B$. Magnetic moment $\mu^{*}$ of $D^{*}$ mesons is estimated from constituent quark magnetic moments $\left(\mu_{c}=(2 / 3) \hbar e / 2 m_{c}=0.4 \mu_{N}\right)$ as $\mu_{D^{0 *}}=\mu_{c}+\mu_{\bar{u}}=-1.45 \mu_{N}$ and $\mu_{D^{+*}}=\mu_{c}+\mu_{\bar{d}}=1.37 \mu_{N}$ (we use $\mu_{u}=1.85 \mu_{N}$ and $\mu_{d}=-0.97 \mu_{N}$ and assume parallel spins of constituent $\left(q^{\uparrow} \bar{q}^{\uparrow}\right)$ pair in $D^{*}(1 \mathrm{~s})$ vector meson ground state).
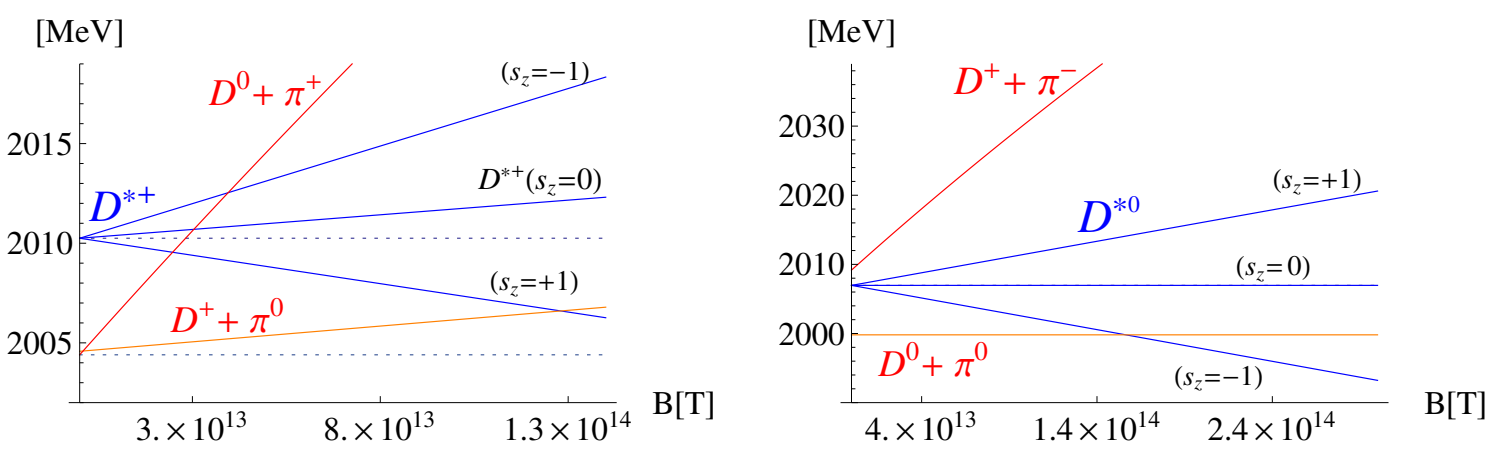

Figure 3: Triplet splitting of $D^{* 0}(2007), D^{*+}(2010)$ vector mesons $\left(\mu_{D^{* 0}} \simeq-1.45 \mu_{N}\right.$ and $\left.\mu_{D^{*+}} \simeq 1.38 \mu_{N}\right)$ in magnetic field $B \approx 10^{14} \mathrm{~T}$, together with the lowest possible energy $\left(p_{z}=0\right)$ of decay products $(D+\pi)$.

One can observe from Fig.3, that decay $D^{*+} \rightarrow D^{0}+\pi^{+}$becomes kinematically forbidden in fields $B \geq 3 \cdot 10^{13} \mathrm{~T}$, and decay $D^{*+} \rightarrow D^{+}+\pi^{0}$ is allowed only for $s_{z}=0,-1$ substates of $D^{+*}$ mesons in fields $B \geq 1.3 \cdot 10^{14} \mathrm{~T}$. Such behavior applies also to $s_{z}=0,+1$ substates of $D^{0 *}$ mesons in fields $B \geq 1.4 \cdot 10^{14} \mathrm{~T}$, and it can result in effective polarisation of decaying $D^{*}$ mesons in magnetic field of relevant strength (assuming here $D^{*}$ mesons were unpolarized).
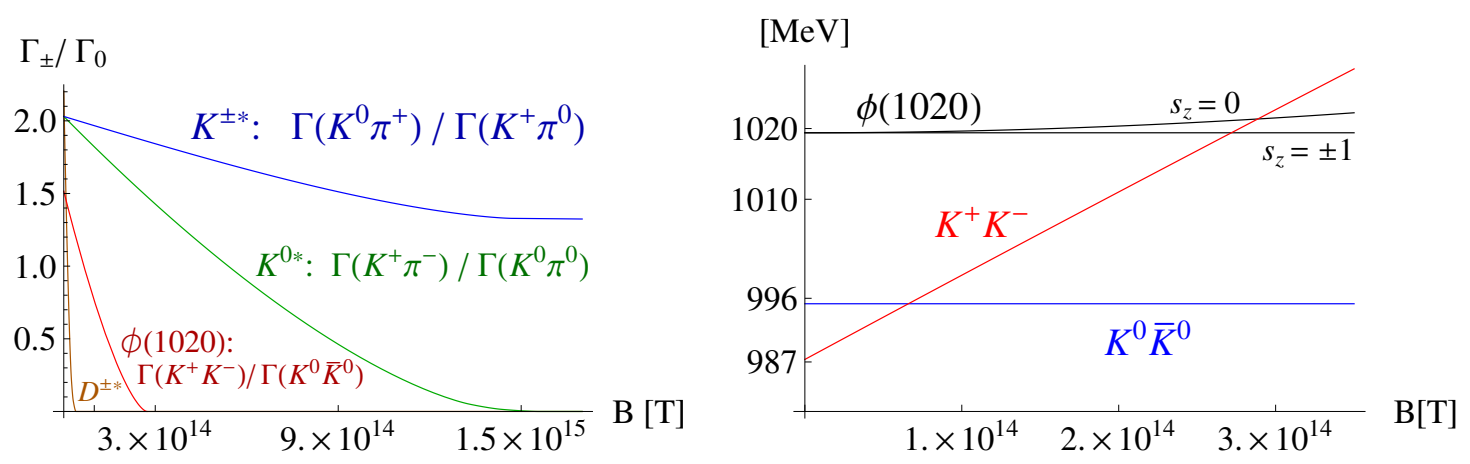

Figure 4: Ratio of decay widths $\Gamma_{ \pm} / \Gamma_{0}$ (charged channel / neutral one = containing $\pi^{0}$ or $K^{0} \bar{K}^{0}$ ) for $K^{ \pm *}$, $K^{0 *}, \phi(1020)$, and $D^{ \pm *}(2010)$ vector mesons in magnetic field (left panel). Energy of $\phi(1020)$ meson (with quadratic Zeeman effect shown) and the lowest possible energy $\left(p_{z}=0\right)$ of $K \bar{K}$ decay products (right panel).

Isospin conservation for strong decays of $D^{*}$ and $K^{*}$ mesons requires ratio of decay widths $\Gamma_{ \pm} / \Gamma_{0}=2$ (here $\Gamma_{0}$ is decay width of channel containing $\pi^{0}$ ). Full isospin violation occurs for $D^{0 *}$ meson (because $M_{D^{0 *}}<M_{D^{+}}+M_{\pi^{-}}$) and magnetic field gradually violates isospin rule in $D^{ \pm *}$ decays. For $K^{*} \rightarrow K+\pi$ decays, modified $\Gamma_{ \pm} / \Gamma_{0}$ ratio (see Figure 4) would introduce systematic bias to extracted $K^{*}(892)$ yields [14] in relativistic heavy ion collision experiments [3]. This is not expected to occur for $D^{ \pm *}$ mesons (lifetime $\tau_{D^{*}}>2000 \mathrm{fm} / \mathrm{c}$ is by factor 500x longer than $\tau_{K^{*}}$ ). 
Behavior of $\phi(1020)$ meson ( $\tau_{\phi} \approx 45 \mathrm{fm} / \mathrm{c}$ in vacuum) is shown in Figure 4. Suppression of $\phi \rightarrow K^{+} K^{-}$decay channel in the magnetic field means longer lifetime of $\phi(1020)$ mesons, which may partially compensate the expected broadening [15] of $\phi \rightarrow K^{+} K^{-}$decay width in dense QCD matter in heavy ion collisions. Our estimate for ratio $\Gamma\left(K^{+} K^{-}\right) / \Gamma\left(K^{0} \bar{K}^{0}\right)$ is shown in Fig.4 (left).

\section{Decay of Baryon Resonances in Magnetic Field}

The phenomenon of partial decay width modification anticipated for vector mesons in magnetic fields may occur also in the case of baryon resonances. For example, the phase-space of decay $\Delta^{++} \rightarrow p+\pi^{+}$can be altered, and channel $\Delta^{0} \rightarrow p+\pi^{-}$may become suppressed in sufficiently strong magnetic fields, as we suggest in Figure 5.
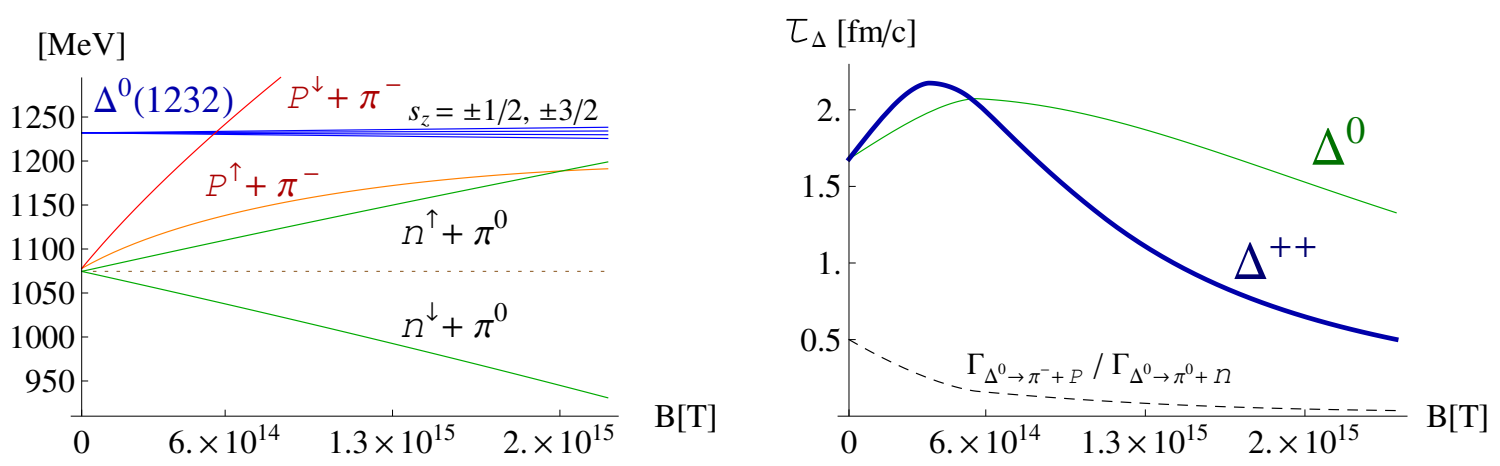

Figure 5: $\Delta^{0}(1232)$ baryon energy (we use $\mu_{\Delta^{0}} \simeq-0.1 \mu_{N}$ ) in magnetic field and the lowest possible energy of decay products $\left(p^{\uparrow \downarrow}, \pi^{-}\right)$and $\left(n^{\uparrow \downarrow}, \pi^{0}\right)$. Right panel shows $\Delta^{0}, \Delta^{++}$lifetimes in magnetic field (see text).

Magnetic interaction of baryon magnetic moment $(\vec{\mu} \cdot \vec{B})$ splits energy of baryons (proton, $\mathrm{n}$ ) into $s_{z}$ quantum substates as shown in Figure 5 (for $\Delta^{0}(1232)$ we use $\mu_{\Delta^{0}}=2 \mu_{d}+\mu_{u}=-0.1 \mu_{N}$, with $\left.\mu_{N}=3.15 \cdot 10^{-14} \mathrm{MeV} / \mathrm{T}\right)$. Partial decay widths $\Gamma_{s_{z}}$ of decay channels $\Delta_{s_{z}}^{0} \rightarrow p^{\uparrow \downarrow}+\pi^{-}$and $\Delta_{s_{2}}^{0} \rightarrow n^{\uparrow \downarrow}+\pi^{0}$ become modified with $p^{3}$ sensitivity given by Equation 2.3 (angular momentum $J=\frac{3}{2}$ of $\Delta(1232)$ forces strong decay products to be created in $P$-wave state). In Figure 5 (right panel) we show our estimate for $\Delta^{++}$and $\Delta^{0}$ lifetimes $\tau_{\Delta}=1 / \Gamma_{\text {tot }}$ evaluated using Eq. 2.3.

Calculated ratio of charged/neutral strong decay widths $\Gamma_{\pi^{-}+P^{\uparrow \downarrow}} / \Gamma_{\pi^{0}+n^{\uparrow \downarrow}}=R_{\Delta}$ for $\Delta^{0}(1232)$ is shown as dashed line in Fig. 5 (right panel). Isospin conservation value $R_{\Delta^{0}}=0.5$ decreases to $R_{\Delta^{0}}=0.16$ at $B=6 \cdot 10^{14} \mathrm{~T}$. Magnetic fields created in relativistic collisions of nuclei may therefore suppress $\Delta^{0} \rightarrow p+\pi^{-}$decays relative to $\Delta^{0} \rightarrow n+\pi^{0}$ channel, and extracted $\Delta^{0}$ yields may thus be underestimated (if isospin violation effect described here is present and not accounted for).

\section{Meson Internal Structure Modification in Electric and Magnetic Fields}

Magnetic field can modify internal quantum state (wave function) of Quarkonium mesons in such way, that bound eigenstates of $(q \bar{q})$ pairs become superpositions of wavefunctions with different $\mathrm{C}$ parity [3]. Similarly to ortho and para-Positronium in magnetic field, one has $[3,16]$

$$
\tilde{\Psi}_{1,0}^{[B]}(n s)=\cos _{\alpha} \Psi_{1,0}(n s)+\sin _{\alpha} \eta_{c}(n s), \quad \tilde{\eta}_{c}^{[B]}(n s)=\cos _{\alpha} \eta_{c}(n s)-\sin _{\alpha} \Psi_{1,0}^{c \bar{c}}(n s)
$$


where mixing parameters $\cos _{\alpha}^{2}+\sin _{\alpha}^{2}=1$ depend on magnetic field [3], and superposition

$$
\tilde{\Psi}_{1,1}^{[E]}(2 s) \approx \Psi_{1,1}(2 s)-\varepsilon_{2}\left(\chi_{c 2}^{0} / \sqrt{6}-\chi_{c 2}^{+2}\right)-\varepsilon_{1} \chi_{c 1}^{0} / \sqrt{2}+\varepsilon_{0} \chi_{c 0}^{0} / \sqrt{3},
$$

becomes the eigenstate of Stark Hamiltonian [16] for Charmonium in electric field. Consequently, two-gluon decays of ortho-Charmonium $\tilde{\Psi}_{1, s_{z}}^{[B, E]}(n s) \rightarrow g g$ become allowed in electric or magnetic field due to $C$ parity-even admixture of $\eta_{c}$ and $\chi_{c}$ states in its wave function. This may decrease lifetimes of $\Psi(n s)$ and $\Upsilon(n s)$ mesons and suppress their $\left(l^{+} l^{-}\right)$decays in sufficiently strong fields.

We suggest that production of $(J=1)$ ortho-Quarkonium mesons in external electromagnetic field can be enhanced, because $(g g \gamma)$ or $(g g g)$ initial state restriction (due to C parity [17]) does not apply to wavefunctions $\tilde{\Psi}_{1, s_{z}}^{[E, B]}(n s)$ and $\tilde{\Upsilon}_{1, s_{z}}^{[E, B]}(n s)$, which are not eigenstates of $\mathrm{C}$ parity.

Observation of unexpectedly large $\left(R_{A A} \approx 7\right)$ enhancement [18] of low- $p_{t} \mathrm{~J} / \Psi$ production in peripheral $\mathrm{Pb}+\mathrm{Pb}$ collisions at $\mathrm{LHC}$ can be directly related to quantum superposition phenomenon we suggest to occur in Quarkonium meson sector, in the presence of external field.

\section{Acknowledgements}

Author is grateful to the organizers of EPS HEP 2015 for the possibility of active participation. This work has been supported by Slovak Grant Agency VEGA under contract N. 2/0197/14.

\section{References}

[1] M.N. Chernodub, Physical Review D82, 085011 (2011).

[2] L.D. Landau and E.M. Lifschitz, Course of Theoretical Physics V. 3 (London: Pergamon Press, 1977).

[3] P. Filip, Journal of Physics: Conf. Series 636, 012013 (2015) [arXiv:1504.07008].

[4] K. Johnson, Acta Physica Polonica B6, 865 (1975).

[5] A. Paulus, D.Vasak, B.Müller, W.Greiner, J. Phys. G: Nucl. Phys. 14, 269 (1988); D.Vasak, K.Wietschorke, B.Müller, W.Greiner, Zeitsch. für Physik C21, 119 (1983).

[6] A. Yaounac, L. Oliver, O. Pene, J.-C. Raynal, Phys. Rev. D8, 2223 (1973).

[7] M. N. Chernodub, Mod. Phys. Lett. A29, 1450162 (2014); also arXiv:1001.0570.

[8] I.-S. Suh and G.J. Mathews, The Astrophysical Journal 546, 1126 (2001).

[9] N.P. Samlos, M. Goldberg and B.T. Meadows, Rev. Mod. Phys. 46, 49 (1974).

[10] G. P. Lepage, Phys. Rev. D42, 3251 (1990).

[11] S. Oneda and E. Takasugi, Phys. Rev. D10, 3113 (1974).

[12] W.-T. Deng and X.-G. Huang, Physical Review C85, 044907 (2012).

[13] C. Amsler et al. (Particle Data Group), Phys. Lett. B667, 1 (2008).

[14] B. Abelev et al. (ALICE collab.), Physical Review C91, 024609 (2015).

[15] F. Klingl, T. Waas, W. Weise, Phys. Lett. B431, 254 (1998).

[16] C.D. Dermer and J.C. Weisheit, Physical Review A40, 5526 (1989).

[17] V.A. Novikov et al., Physics Reports 41C, 1 (1978).

[18] A. Lardeux (on behalf of ALICE coll.), presentation N.149, at Hard Probes 2015 conference, Montréal, June $29^{\text {th }}, 2015$, to be published in Nuclear Physics B Proceedings Supplement. 